\title{
CONSTRUCTIVE EXISTENCE RESULTS FOR SOLUTIONS TO SYSTEMS OF BOUNDARY VALUE PROBLEMS VIA GENERAL LYAPUNOV METHODS
}

\author{
J. Henderson, Q. Sheng And C. C. Tisdell
}

\begin{abstract}
In this work we consider boundary value problems (BVPs) for systems of secondorder, ordinary differential equations. A priori bounds on solutions are obtained via differential inequalities involving general Lyapunov functions without the need for maximum principles. These bounds are then applied to produce new existence theorems via topological methods. Some constructive results are also developed via A-proper mappings and the Galerkin method, in which solutions to the BVP may be approximated.
\end{abstract}

Mathematics subject classification (2010): 34B15.

Keywords and phrases: Boundary value problem, ordinary differential equation, existence of solutions, differential inequality, Lyapunov functions, systems of equations.

\section{REFERENCES}

[1] S. R. Bernfeld, G. S. Ladde And V. Lakshmikantham, Existence of solutions of two point boundary value problems for nonlinear systems, J. Differential Equations, 18, (1975), 103-110.

[2] P. W. Eloe, Y. N. RafFoul AND C. C. Tisdell, Existence, uniqueness and constructive results for delay differential equations, Electron. J. Differential Equations. 2005 (2005), no. 121, pp. 1-11.

[3] R. E. Gaines And J. Mawhin, Coincidence Degree and Nonlinear Differential Equations, Lecture Notes in Mathematics, 568, Springer-Verlag, Berlin-New York, 1977.

[4] P. HARTMAN, On two-point boundary value problems for nonlinear second order systems, SIAM J. Math. Anal., 5, (1974), 172-177.

[5] P. Hartman, Ordinary Differential Equations, corrected reprint of the second (1982) edition, Classics in Applied Mathematics, 38, Society for Industrial and Applied Mathematics (SIAM), Philadelphia, PA, 2002.

[6] P. HaRTMAn, On boundary value problems for systems of ordinary, nonlinear, second order differential equations, Trans. Amer. Math. Soc., 96, (1960), 493-509.

[7] K. A. Heimes, Boundary value problems for ordinary nonlinear second order systems, J. Differential Equations, 2, (1966), 449-463.

[8] N. G. Lloyd, Degree Theory, Cambridge Tracts in Mathematics, 73, Cambridge University Press, Cambridge-New York-Melbourne, 1978.

[9] A. LASOTA AND J. A. YORKE, Existence of solutions of two-point boundary value problems for nonlinear systems, J. Differential Equations 11, (1972), 509-518.

[10] W. V. Petryshyn, Solvability of various boundary value problems for the equation $x^{\prime \prime}=$ $f\left(t, x, x^{\prime}, x^{\prime \prime}\right)-y$, Pacific J. Math., 122, (1986), 169-195.

[11] W. V. PETRYSHYN, Existence theorems for semilinear abstract and differential equations with noninvertible linear parts and noncompact perturbations, Nonlinear equations in abstract spaces (Proc. Internat. Sympos., Univ. Texas, Arlington, Tex., 1977), pp. 275-316, Academic Press, New York, 1978.

[12] K. Schmitt AND R. Thompson, Boundary value problems for infinite systems of second-order differential equations, J. Differential Equations, 18, (1975), 277-295. 
[13] C. C. TISDEll AND M. Holzer, Analysis of the boundary value problem associated with the nonrelativistic Thomas-Fermi equation for heavy atoms in intense magnetic fields, Differ. Equ. Appl., 7, (2015), no. 1, 27-41. 